\title{
BAYESIAN COMPUTATIONAL METHODS FOR SPATIAL ANALYSIS OF IMAGES
}

\author{
MATTHEW T. MOORES
}

(Received 10 November 2015; first published online 8 January 2016)

2010 Mathematics subject classification: primary 62F15; secondary 62P10, 62H35.

Keywords and phrases: Bayesian inference, cone-beam computed tomography, image segmentation, Potts/Ising model, approximate Bayesian computation.

This thesis introduces a novel representation for prior information in a spatial model and develops scalable algorithms for fitting this model to large imaging datasets. These methods are employed for image-guided radiation therapy and satellite-based classification of land use and water quality. This study has utilised a pre-computation step to achieve a substantial improvement in the elapsed runtime for model fitting. This makes it much more feasible to apply these models to real-world problems, and enables full Bayesian inference for images with a million or more pixels.

Our research addresses an important applied problem in image-guided radiation therapy, as part of an interdisciplinary collaboration between Queensland University of Technology and the Radiation Oncology Mater Centre, Queensland Health. The aim is to assist medical practitioners in interpreting cone-beam computed tomography (CT) of radiotherapy patients by labelling the image pixels according to tissue type. These medical images have poor contrast-to-noise ratio (CNR); thus external sources of information are needed for accurate segmentation. Such sources include the individualised treatment plan, which is based on a diagnostic-quality CT scan that has been manually labelled by a clinician. We also use published studies of physiological variability to derive an estimate of spatial uncertainty.

To address this problem, we adopt a hidden Potts model of the image lattice. The Potts model [6] is a discrete Markov random field that can be used to label the pixels in an image according to an unobserved classification. In [3] we introduce a method for deriving and representing the spatial prior for each patient as an external field in the hidden Potts model. This method is applied to cone-beam CT scans of an electron density (ED) phantom, to test the method on images where the true segmentation

Thesis submitted to Queensland University of Technology in February 2015; degree approved on 11 June 2015; supervisors: Kerrie Mengersen and Fiona Harden.

(c) 2016 Australian Mathematical Publishing Association Inc. 0004-9727/2016 \$16.00 
is known. We apply our method to radiotherapy patient data in [2]. Tissue density estimates are derived from the planning CT and adjusted to account for differences in image modality, forming priors for the noise parameters. These priors can be updated sequentially as more images of the patient are acquired.

The strength of spatial dependence between neighbouring labels is governed by the inverse temperature parameter of the Potts model. This parameter is difficult to estimate, due to its dependence on an intractable normalising constant. Several approaches have been proposed, including the exchange algorithm and approximate Bayesian computation (ABC). Our comparative study [5] demonstrates that algorithms that rely on simulating auxiliary variables at every iteration are too computationally intensive for use in large-scale image analysis. We introduce a pre-computation step in [1] that fits an auxiliary model to map from the inverse temperature to its sufficient statistic. With this auxiliary model, we achieve an improvement of two orders of magnitude in the scalability of approximate Bayesian computation with sequential Monte Carlo (ABC-SMC). Our implementations of these algorithms are incorporated into an open source library for the R software platform [4].

\section{Acknowledgements}

I gratefully acknowledge the financial support of QUT and the Australian federal government Department of Education, Science and Training. Computational resources and services used in this work were provided by the HPC and Research Support Group, QUT. Portions of this thesis were completed during academic visits to the Norwegian University of Science and Technology (NTNU), Université Paris Dauphine and University of Warwick.

\section{References}

[1] M. T. Moores, C. C. Drovandi, K. Mengersen and C. P. Robert, 'Pre-processing for approximate Bayesian computation in image analysis', Stat. Comput. 25(1) (2015), 23-33.

[2] M. T. Moores, C. E. Hargrave, T. Deegan, M. Poulsen, F. Harden and K. Mengersen, 'An external field prior for the hidden Potts model with application to cone-beam computed tomography', Comput. Statist. Data Anal. 86 (2015), 27-41.

[3] M. T. Moores, C. E. Hargrave, F. Harden and K. Mengersen, 'Segmentation of cone-beam CT using a hidden Markov random field with informative priors', Proc. 17th Int. Conf. ICCR, J. Phys.: Conf. Ser., Vol. 489 (Melbourne, Australia, 2014), 012076.

[4] M. T. Moores and K. Mengersen, 'bayesImageS: Bayesian methods for image segmentation using a hidden Potts model', R package version 0.1-21, 2015; http://researchdatafinder.qut.edu.au/ display/n11957.

[5] M. T. Moores, A. N. Pettitt and K. Mengersen, 'Scalable Bayesian inference for the inverse temperature of a hidden Potts model', Preprint, 2015, arXiv:1503.08066; http://arxiv.org/abs/1503.08066.

[6] R. B. Potts, 'Some generalized order-disorder transformations', Proc. Cambridge Philos. Soc. 48(1) (1952), 106-109.

\section{MATTHEW T. MOORES, University of Warwick, Coventry CV4 7AL, UK e-mail: M.T.Moores@warwick.ac.uk}

